\title{
The effect of fixation plate use on bone healing during the reconstruction of mandibular defects
}

\author{
Khang Do Gia Hong, Seong-Gon Kim, Young-Wook Park \\ Department of Oral and Maxillofacial Surgery, College of Dentistry, Gangneung-Wonju National University, Gangneung, Korea
}

\begin{abstract}
J Korean Assoc Oral Maxillofac Surg 2019;45:276-284)
Objectives: This study sought to compare efficiency results between the use of a customized implant (CI) and a reconstruction plate (RP) in mandibular defect reconstruction in an animal model.

Materials and Methods: Fifteen rabbits underwent surgery to create a defect in the right side of the mandible and were randomly divided into two groups. For reconstruction of the mandibular defect, the RP group $(n=5)$ received five-hole mini-plates without bone grafting and the CI group ( $n=10$ ) received fabricated CIs based on the cone-beam computed tomography ( $\mathrm{CBCT}$ ) data taken preoperatively. The CI group was further divided into two subgroups depending on the time of CBCT performance preoperatively, as follows: a six-week CI (6WCI) group ( $\mathrm{n}=5)$ and a one-week CI (1WCI) group $(n=5)$. Daily food intake amount (DFIA) was measured to assess the recovery rate. Radiographic images were acquired to evaluate screw quantity. CBCT and histological examination were performed in the CI subgroup after sacrifice.

Results: The 1WCI group showed the highest value in peak average recovery rate and the fastest average recovery rate. In terms of reaching a $50 \%$ recovery rate, the $1 \mathrm{WCI}$ group required the least number of days as compared with the other groups (2.6 \pm 1.3 days), while the RP group required the least number of days to reach an $80 \%$ recovery rate (7.8 \pm 2.2 days). The $1 \mathrm{WCI}$ group showed the highest percentage of intact screws $(94.3 \%)$. New bone formation was observed in the CI group during histological examination.

Conclusion: Rabbits with mandibular defects treated with CI showed higher and faster recovery rates and more favorable screw status as compared with those treated with a five-hole mini-plate without bone graft.
\end{abstract}

Key words: Mandibular reconstruction, Mandibular defect, 3D printing, Customized implant, Reconstruction plate

[paper submitted 2019. 3. 13 / revised 1st 2019. 5. 10, 2nd 2019. 5. 29 / accepted 2019. 6. 3]

\section{Introduction}

The mandible is the most significant and most durable bone of the skull. Its position is at the lowest point of the human face, beneath the maxilla. Aside from the ossicles of the middle ear, the mandible is the second movable bone of the skull $^{1}$. The mandible is essential to the formation and function of the oral cavity. Its primary function is to form the lower jaw and hold the lower teeth in place. It also provides the

\footnotetext{
Young-Wook Park

Department of Oral and Maxillofacial Surgery, College of Dentistry, Gangneung-Wonju National University, 7 Jukheon-gil, Gangneung 25457, Korea

TEL: +82-33-640-3183 FAX: +82-33-640-3103

E-mail:ywpark@gwnu.ac.kr

ORCID: https://orcid.org/0000-0001-5881-7257

(a) This is an open-access article distributed under the terms of the Creative Commons Attribution Non-Commercial License (http://creativecommons.org/ licenses/by-nc/4.0/), which permits unrestricted non-commercial use, distribution and reproduction in any medium, provided the original work is properly cited.

Copyright (C) 2019 The Korean Association of Oral and Maxillofacial Surgeons. All rights reserved.
}

support and foundation for tongue positioning and elevation of the larynx during swallowing ${ }^{2}$. Mandibular continuity defects that result from trauma, congenital deformity, or tumor resection have an impact on patients' physical function and aesthetics ${ }^{2}$. For functional rehabilitation of affected patients, the continuity defect must be restored immediately or as soon as possible ${ }^{3}$.

Through the course of history, many options for reconstruction after mandibular defect have been introduced, such as free bone grafts, vascularized rib, or alloplasts; however, these often did not result in optimal outcomes as expected ${ }^{4,5}$. In the mid-1970s, vascularized mandibular reconstruction was introduced and, since then, has become the standard protocol for the treatment of significant defects of the jaw ${ }^{6}$. The fibular free flap has become the most popular flap for mandible reconstruction because it can provide excellent bone stock with reliable anatomy, and the flap can be harvested using a two-team approach ${ }^{7}$. However, the morbidity of the donor site, a lengthy operation, and suboptimal aesthetics results are 
possible disadvantages of using this type of flap ${ }^{8,9}$. Another factor that should be considered is that in situations where the mandibular defect is due to the recession of a tumor, caution when choosing to perform vascularized free flap as the primary reconstruction protocol is warranted because of a high recurrence rate ${ }^{10}$. This approach should only be performed when no recurrence of the lesion is confirmed through oral and radiographic examinations.

The introduction of the metallic reconstruction plate (RP) in the 1980s provided an alternative treatment protocol for primary reconstruction of a mandibular defect. Such could be used to achieve stable fixation of free bone grafts and soft tissue reconstruction ${ }^{11}$. Another purpose of the RP is to bridge and maintain the position of the two free bone segments of a resected mandible, providing an adequate condition for subsequent reconstruction using a vascularized free flap if there is no recurrence of the lesion ${ }^{12}$. Although this technique has become popular in conjunction with vascularized free flap, a variety of complications including plate fracture, plate exposure, screw loosening, and infection through the mucosa or skin have been observed ${ }^{13,14}$. A key reason for the failure of the RP system is most likely the high force created during masticatory movement of the patient ${ }^{15}$. Moreover, in cases with significant mandibular defects or when the defect is adjacent to/involves the condyle, placement of a long RP with limited to no space for screw fixation could prove to be inadequate for primary reconstruction. Consequently, a proposal for a new reconstruction option must be suggested.

Cone-beam computed tomography (CBCT), in combination with computer-aided design (CAD)/computer-aided manufacturing (CAM), has been previously used by clinicians to set up thorough and precise treatment plans for reconstruction surgeries. Throughout this process, a plastic model of the patient is made from the CBCT data. A surgical guide is then fabricated by $\mathrm{CAD} / \mathrm{CAM}$ for assisting the clinician during the osteotomy procedure ${ }^{16}$. On the other hand, CAD/CAM technology has the advantages of creating a digital reconstruction of unilateral mandibular defects based on the anatomy characteristics of the unaffected side ${ }^{17}$. This idea involves the use of CAD/CAM to produce a customized implant (CI) for reconstruction of the mandibular defect of a specific patient. However, CIs for mandibular defects are difficult to design and produce because of the requirement to withstand high mechanical stress. Recently, there was a case report published that described a successful reconstruction of a mandibular defect using a CI produced by the combination of $\mathrm{CAD} / \mathrm{CAM}$ technology and three-dimensional (3D) print- ing by electron-beam melting ${ }^{18}$. Although this could prove to be a promising treatment method for the reconstruction of mandibular defects, comparative studies between this new protocol and conventional protocols, especially in an animal model, are needed before it can be widely approved for use in humans.

In this study, mandibular defects were created surgically in a rabbit model. Because of the relatively small size of the rabbit's mandible as compared with the human's, five-hole titanium mini-plates without any bone graft were used as a RP system to reconstruct the defects in the control group, and CI by $3 \mathrm{D}$ printing was used to restore the deficiencies in the experimental groups. The aims of this study were to compare the efficiency of CI and RP in the reconstruction of mandibular defect in an animal model in regard to recovery indicated by daily food intake amount (DFIA) and screw status.

\section{Materials and Methods}

\section{Implant fabrication}

Pure titanium grade 1 spherical powder (average particle size, $35 \mathrm{~mm}$ ) was used as a base material for 3D printing using the selective laser melting (SLM) method with the Realizer SLM300i system (Realizer, Borchen, Germany). Purified argon gas was then pumped into the chamber to prevent oxidation of the material. The CI was printed in a layer-tolayer pattern, with each layer being $25 \mathrm{~mm}$ thick. In terms of the processing parameters, a laser power of $200 \mathrm{~W}$ and a scan speed of $500 \mathrm{~mm} / \mathrm{s}$ were applied. After the CI was printed, residual titanium powder was removed by using an ultrasonic cleaner.

\section{Animal experiment}

This experiment was approved by the Institutional Animal Care and Use Committee of Gangneung-Wonju National University, Gangneung, Republic of Korea (GWNU-201625). Fifteen New Zealand white rabbits were used for this study and were randomly assigned to one of two groups. The RP group (control group, $n=5$ ) received a five-hole mini-plate without any bone graft for reconstruction of the mandibular defect, while the CI group (experimental group, $\mathrm{n}=10$ ) had their defect reconstructed by use of a CI instead. The CI group was then divided into two subgroups, a six-week CI (6WCI) group and a one-week CI (1WCI) group. The difference between these two subgroups was the amount of time 
from CI fabrication to the reconstruction surgery (six weeks vs one week). The $6 \mathrm{WCI}$ group used the CBCT data taken at six weeks before surgery for the manufacturing of the CI, while the CBCT data for the 1WCI group was taken at one week before the surgery.(Fig. 1)

In consideration of the mandibular defect, a bony segment measuring $5 \mathrm{~mm}$ in the right side of the mandibular body, between the molar and incisor, was chosen to be removed surgically to create the defect. A CBCT scan was performed on each rabbit, and surgical guides for the osteotomy procedure were manufactured using $\mathrm{CAD} / \mathrm{CAM}$ technology. The CIs were then designed based on the individual CBCT data of each rabbit in the CI group. The printing process for the CI was already described in the previous section. The internal structure of the implant was designed to be as similar to the porous structure of original bone as possible, and the pore size was 800 mm.(Fig. 2)

The operation was performed with the rabbits under druginduced general anesthesia by an intramuscular injection of a combination of Zoletil 50 (15 mg/kg; Vibac Korea, Seoul, Korea) and Rompun $(0.2 \mathrm{~mL} / \mathrm{kg}$; Bayer Korea, Seoul, Korea). Each rabbit was then injected with gentamicin $(0.1 \mathrm{~mL} /$ kg; Samu Median, Seoul, Korea) and tolfenamic acid (0.1 $\mathrm{mL} / \mathrm{kg}$; Samyang Anipharm, Seoul, Korea) intramuscularly as preoperation medication.

After the hair had been shaved, the right mandibular area was disinfected with iodine solution and local anesthesia was administered. A submandibular incision on the right side of the mandible was performed and the skin and platysma muscle were incised while ensuring hemostasis. After exposure of the mandibular periosteum, a periosteal incision and subperiosteal dissection were performed. The right side of the mandibular body was exposed, and a surgical guide was used to mark the recession margin before vertical osteotomy. The defect was then created by the use of a reciprocating saw. Each mandibular defect was then reconstructed using the appropriate protocol depending on the group. For the RP group, a five-hole titanium mini-plate (MN/P SB 14MM 1.0T G3; OsteoMed, Dallas, TX, USA) and four mini-screws (20-MN006 and 20-MN-008, diameter: $2.0 \mathrm{~mm}$ and length: $6.0 \mathrm{~mm}$; Jeil Medical Corporation, Seoul, Korea) were used for fixation and reconstruction of the mandibular defect.(Fig. 3) Each rabbit of the CI group received their specific indicated CI for reconstruction, and micro-screws were used for the fixation of each implant.(Fig. 4) After review of the final positioning of the plates or CIs, closure by layer was performed using 4-0 Vicryl sutures (Johnson \& Johnson, New Brunswick, NJ, USA). Gentamicin and tolfenamic acid were then injected intramuscularly after the operation.

Each rabbit was individually caged and received Purina Rabbit Chow (Purina Mills, Gray Summit, MO, USA) as food as well as water without any shortage. The functional recovery rate was assessed by measuring the daily food intake amount $^{19}$, as calculated by the ratio between DFIA for each
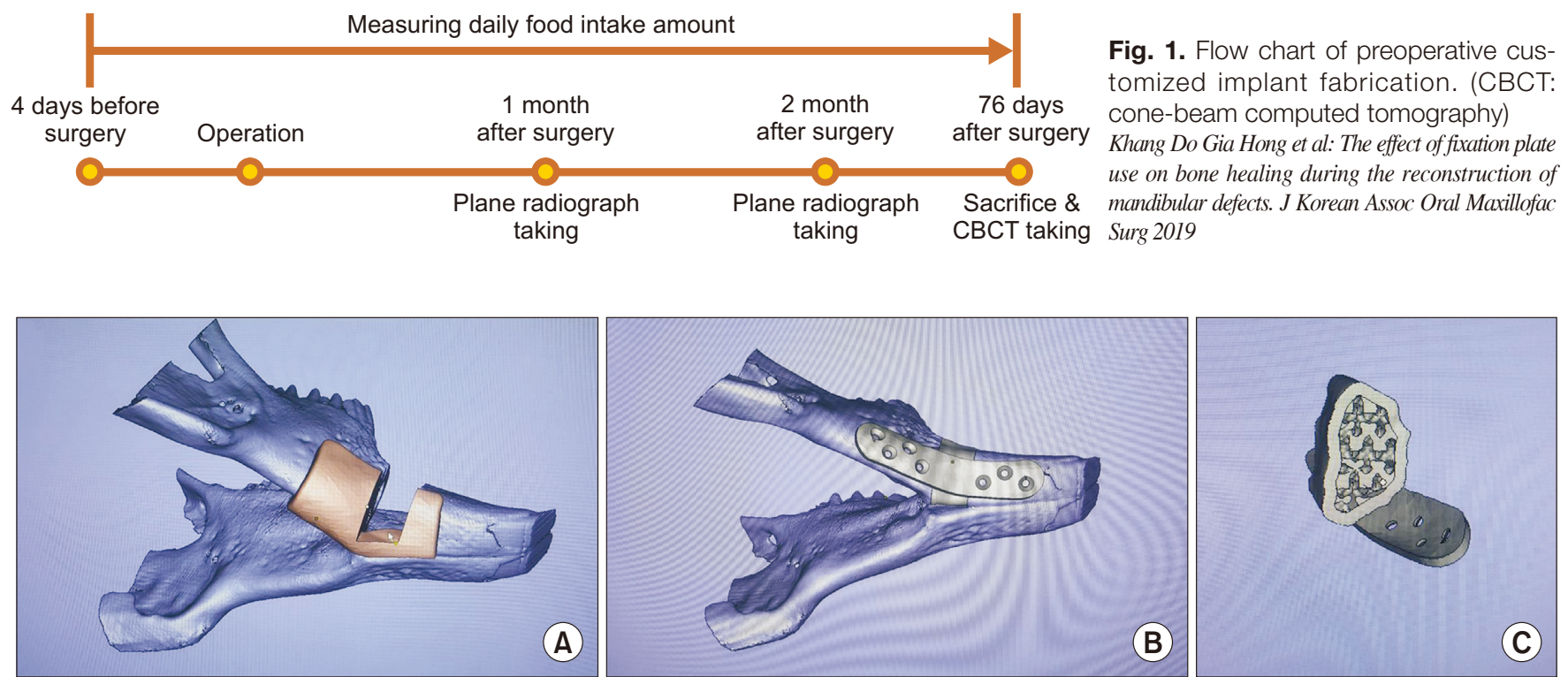

Fig. 2. A. Individual computed tomography (CT) scans of rabbits were used for designing surgical guide. B. Customized implant (Cl) was designed using individual CT scans based on preplanned mandibular defects. C. The cross section of a Cl mimicked the internal porous structure of the mandible.

Khang Do Gia Hong et al: The effect of fixation plate use on bone healing during the reconstruction of mandibular defects. J Korean Assoc Oral Maxillofac Surg 2019 

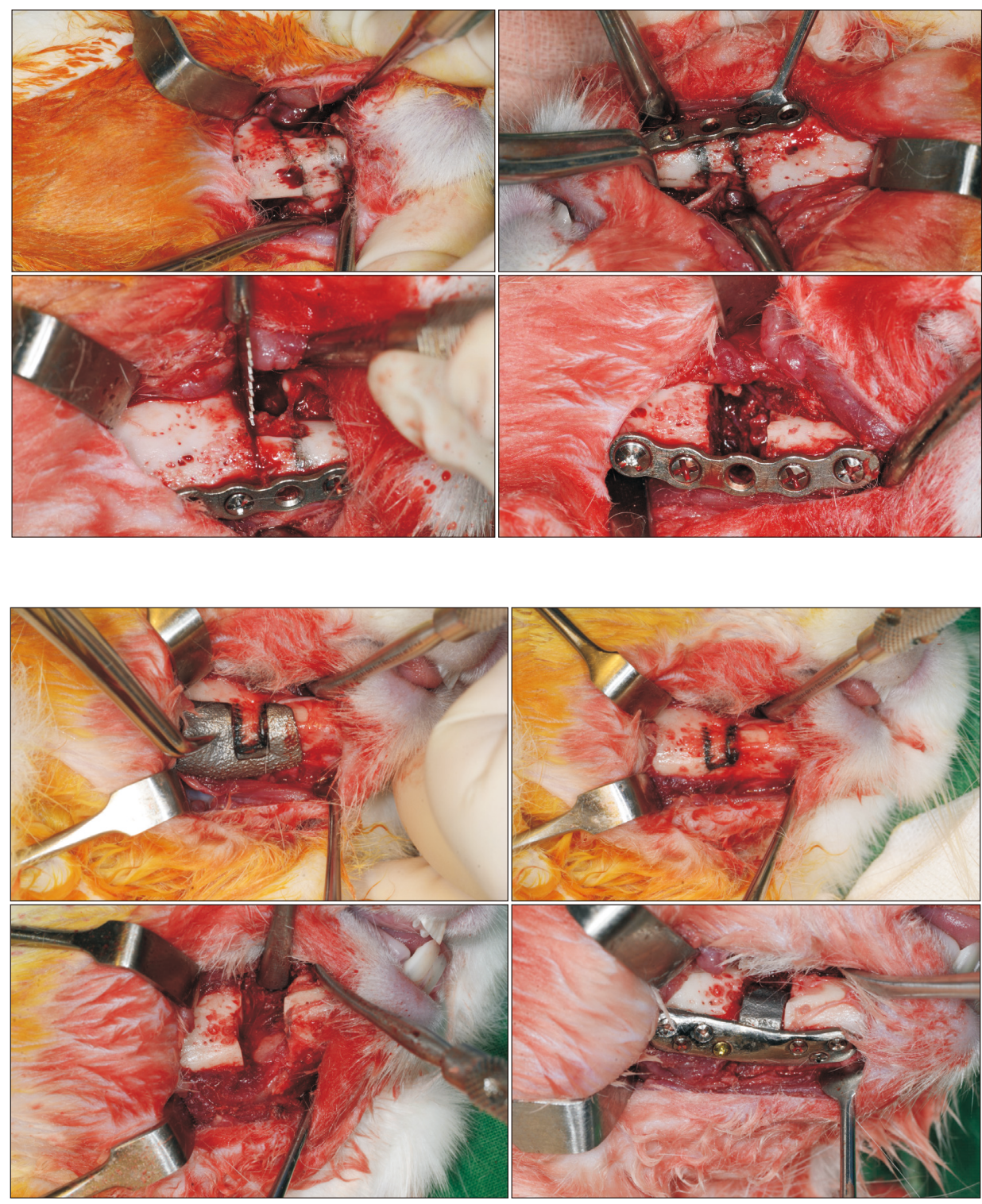

Fig. 3. Marking of the recession margin was made by temporary fixation of the reconstruction plate. The defect was then created by the use of a reciprocating saw. Final fixation of the plate was performed.

Khang Do Gia Hong et al: The effect of fixation plate use on bone healing during the reconstruction of mandibular defects. J Korean Assoc Oral Maxillofac Surg 2019

Fig. 4. The surgical guide was used to mark the recession margin before vertical osteotomy. The defect was then created by the use of a reciprocating saw. Customized implant $(\mathrm{Cl})$ with micro-screws was used for fixation of each implant in $\mathrm{Cl}$ group.

Khang Do Gia Hong et al: The effect of fixation plate use on bone healing during the reconstruction of mandibular defects. J Korean Assoc Oral Maxillofac Surg 2019 day postoperation and preoperative average DFIA (Equation 1). Individually, DFIA was measured for four days before the surgery and for 76 days postoperation.

Daily recovery rate $(\%)=$

Daily food intake amount Average daily food intake amount preoperation $\times 100$

Plain radiographic images (parallel technique) were obtained for each rabbit immediately postoperation and at monthly intervals thereafter using the X-ray generator HEL$\mathrm{S}$ (Shinhung, Seoul, Korea) with 0.10 seconds of exposure time. The radiographic results were used to evaluated screw quantity between each time interval. All rabbits were then sacrificed humanely at the end of the observation period.
During the sacrifice procedure, screw status and the presence of new bony ingrowth into the CI pores were evaluated. CBCT scans of each rabbit head were then taken post sacrifice for further evaluation of screw loosening based on changes in the positioning of the screw thread as compared with the appearance on the immediate postoperative plain radiographs. Screws that were entirely lost during the observation period were also be classified as being loosened for the purpose of this study. The screw status between groups was compared based on the percentage of screws that were intact versus loosened.

For the histologic analysis, the middle of the CI between the edges of the bony defect in each rabbit was crosssectioned. Sectioned specimens were embedded in lightcuring resin after a gradual dehydration process. Polymerized 
specimens were ground and polished ${ }^{20}$. The final thickness of the sliced specimens was $2 \mathrm{~mm}$. Sections were stained with Goldner trichrome stain and examined under a light microscope.

\section{Statistical analysis}

The one-way ANOVA test was used to compare DFIA values between the RP, 6WCI, and 1WCI groups. If statistical significance was found, a post-hoc test was performed to compare each group to the other two groups. Tukey's test was chosen to be the post-hoc test and the statistical significance was set at $P<0.05$. The statistical analysis was performed using the IBM SPSS Statistics software program (ver. 25; IBM Corp., Armonk, NY, USA).

\section{Results}

The average recovery rates of all groups are shown in Fig. 5. A similar pattern was observed in all groups. After reaching the peak average recovery rate after approximately three weeks, the rabbits maintained stable values for two months and then started to decline gradually until the end of the observation period. In terms of both the peak average recovery rate and the fastest average recovery rate, the $1 \mathrm{WCI}$ group showed the highest values out of all of the groups. The average time periods required to achieve $50 \%$ and $80 \%$ recovery for each rabbit in each group are presented in Fig. 6. The $1 \mathrm{WCI}$ group required $2.6 \pm 1.3$ days and $8.2 \pm 3.3$ days to reach $50 \%$ and $80 \%$ recovery, respectively. The $6 \mathrm{WCI}$ group required $7.8 \pm 2.3$ days and $10.8 \pm 1.8$ days to reach $50 \%$ and $80 \%$ recovery, respectively. Finally, the RP group required $4.6 \pm 1.5$ days and $7.8 \pm 2.2$ days to reach $50 \%$ and $80 \%$ recovery, respectively.

The one-way ANOVA test showed statistically significant differences between the average days required for $50 \%$ recovery of the $6 \mathrm{WCI}, 1 \mathrm{WCI}$, and RP groups $(P=0.002)$. However, there were no statistically significant differences between the average days required for $80 \%$ recovery among the groups. More specifically, there was a statistical difference between the 6WCI group and RP group at 50\% recovery ( $P=0.035$ ), while the difference between the 6WCI group and

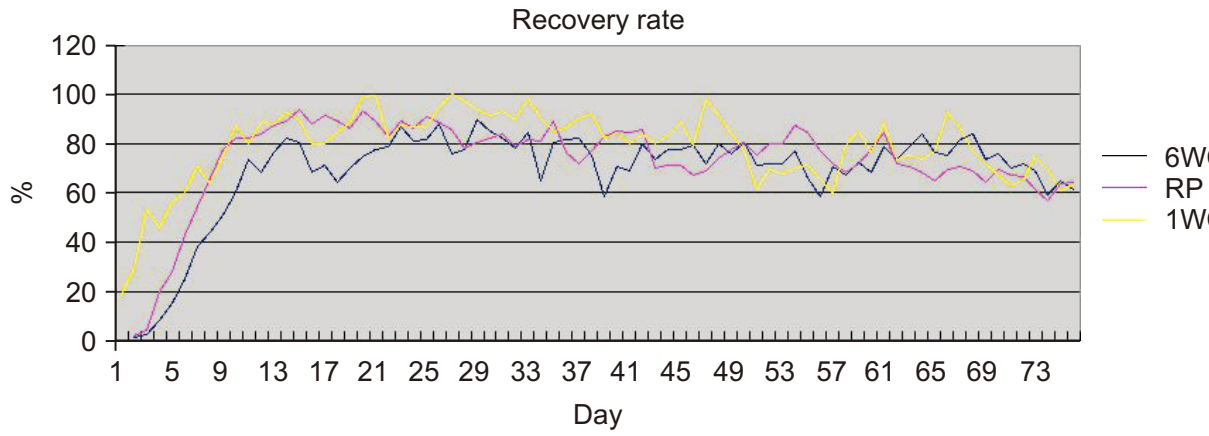

Fig. 5. Average recovery rate (\%) of each groups. (6WCl: six-week customized implant, RP: reconstruction plate, 1WCl: one-week customized implant) Khang Do Gia Hong et al: The effect of fixation plate use on bone healing during the reconstruction of mandibular defects. J Korean Assoc Oral Maxillofac Surg 2019
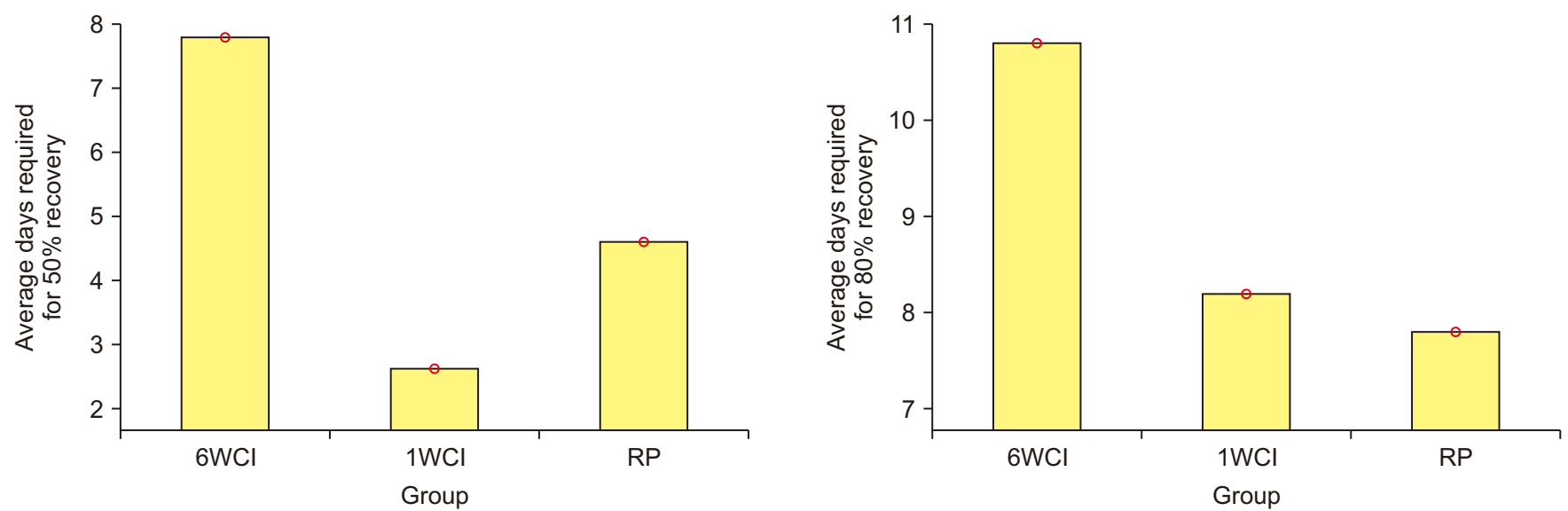

Fig. 6. Days required to reach $50 \%$ and $80 \%$ recovery rate in each individuals. (6WCl: six-week customized implant, $1 \mathrm{WCl}$ : one-week customized implant, RP: reconstruction plate)

Khang Do Gia Hong et al: The effect of fixation plate use on bone healing during the reconstruction of mandibular defects. J Korean Assoc Oral Maxillofac Surg 2019 
$1 \mathrm{WCI}$ group was also statistically different at 50\% recovery $(P=0.001)$. However, there was no statistical difference between the $1 \mathrm{WCI}$ group and RP group at $50 \%$ recovery. During the observation period, all of the rabbits achieved a recovery rate of more than $90 \%$.

The numbers of screws (35 and 20, respectively) in the $1 \mathrm{WCI}$ group and RP group did not change during the observation time. In the $6 \mathrm{WCI}$ group, at both the one-month and two-month intervals, the remaining screws numbered 28 $(84.9 \%)$, while, at the point of sacrifice, there were 26 screws present in the 6WCI group (78.8\%), for a total of seven screws lost during the observation time in this group. Of all groups, the 1WCI group maintained the highest percentage of intact screws (94.3\%). The percentages of intact screws in the 6WCI group and RP group were $75.8 \%$ and $85.0 \%$, respectively.(Table 1)

During the observation time, there was one rabbit in the 6WCI group that showed a complication of wound infection with CI exposure at one month postoperation, while another rabbit in the same group presented a similar condition at two months postoperation. Both were treated with the same medication as was given immediately postoperation for three

Table 1. Screw status at sacrifice in each group

\begin{tabular}{lcl}
\hline & Intact & Loosened $^{1}$ \\
\hline 6WCI & $25 / 33(75.8)$ & $8 / 33(24.2)$ \\
1WCI & $33 / 35(94.3)$ & $2 / 35(5.7)$ \\
Reconstruction plate & $17 / 20(85.0)$ & $3 / 20(15.0)$ \\
\hline
\end{tabular}

(6WCI: six-week customized implant, $1 \mathrm{WCI}$ : one-week customized implant)

${ }^{1}$ Loosened screws include screws that were fully lost as well.

Khang Do Gia Hong et al: The effect of fixation plate use on bone healing during the reconstruction of mandibular defects. J Korean Assoc Oral Maxillofac Surg 2019

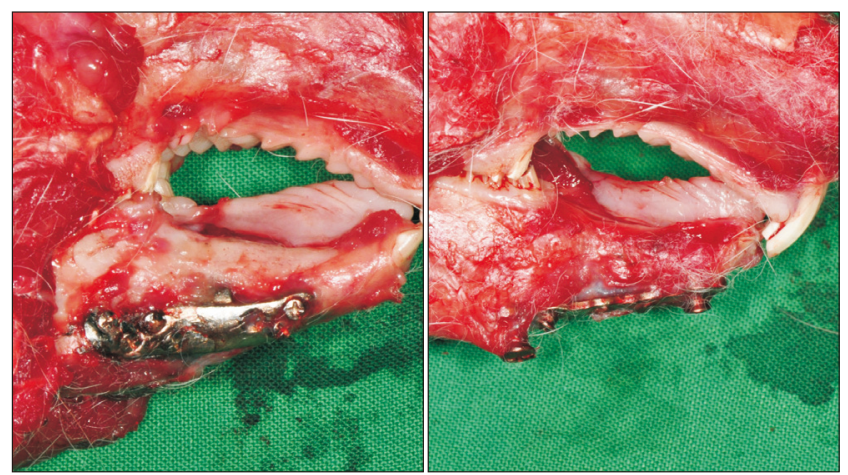

Fig. 7. The customized implant $(\mathrm{Cl})$ sample show bone overgrowth the main body of the implant with no screw loosening while there was sign of screw loosening on the reconstruction plate sample.

Khang Do Gia Hong et al: The effect of fixation plate use on bone healing during the reconstruction of mandibular defects. J Korean Assoc Oral Maxillofac Surg 2019 days and daily dressing using iodine solution. At the end of the observation period, both showed complete healing of their secondary wounds. The other rabbits showed no sign of postoperative complications. At sacrifice, only one rabbit in the 6WCI group demonstrated instability of the CI, and no evidence of new bone generation was found in this rabbit. All other CIs and plates were stable at the time of sacrifice.(Fig. 7 ) In general, the rates of CI exposure and unstable CI in the $6 \mathrm{WCI}$ group were $40 \%$ and $20 \%$, respectively.

According to the CBCT data after the sacrifice, there was confirmation of successful new bone generation in the rabbits of the CI group with no loosened screws.(Fig. 8. A) In the case of a rabbit that had one loosened screw at sacrifice, a lower level of bony regeneration was still observed in the margin area around the CI.(Fig. 8. B) Histologic examination of the rabbits with no loosened screws showed evidence of bony ingrowth into the pores structure of the titanium CI structure.(Fig. 9. A) In the case of the rabbit with plate exposure and unstable $\mathrm{CI}$, bony ingrowth into the CI was not
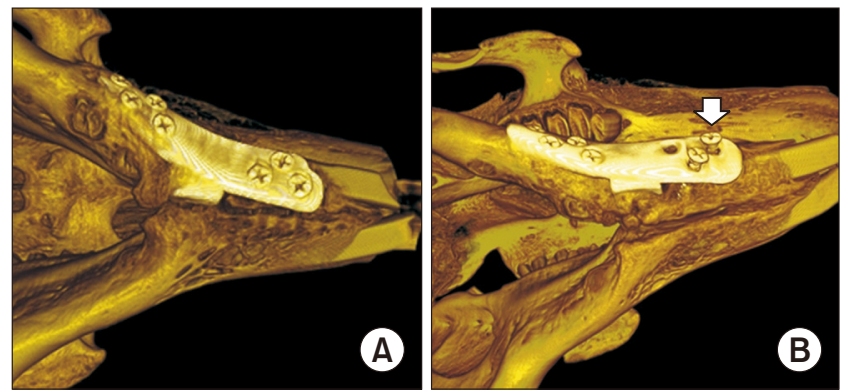

Fig. 8. Reconstructed images based on computed tomography scans after sacrifice. A. Rabbit with no loosened screws. B. Rabbit with loosened screws (arrow).

Khang Do Gia Hong et al: The effect of fixation plate use on bone healing during the reconstruction of mandibular defects. J Korean Assoc Oral Maxillofac Surg 2019
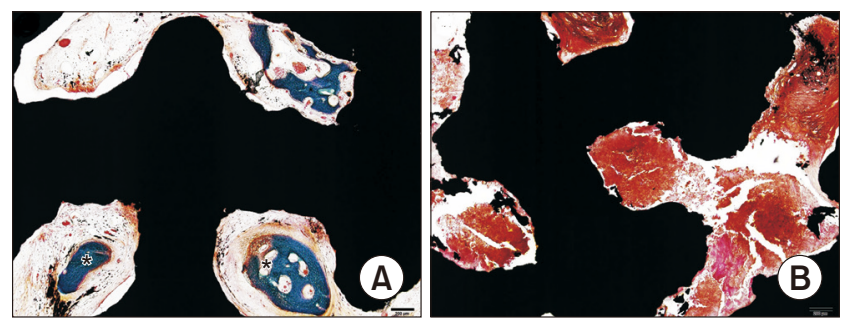

Fig. 9. A. Histologic examination showed evidence of bony ingrowth (asterisks) into the porous structure of the customized implant $(\mathrm{Cl})$ in the rabbit with no loosened screws. B. In the case of the animal with plate exposure and unstable $\mathrm{Cl}$, bony ingrowth into the $\mathrm{Cl}$ was not observed. A, B. Goldner trichrome stain. Scale bars $=200 \mu \mathrm{m}$.

Khang Do Gia Hong et al: The effect of fixation plate use on bone healing during the reconstruction of mandibular defects. J Korean Assoc Oral Maxillofac Surg 2019 
observed.(Fig. 9. B)

\section{Discussion}

In this study, DFIA was used as a primary indicator to demonstrate the recovery rate of each rabbit ${ }^{21,22}$. As shown in Fig. 5, the 1WCI group achieved the highest value in terms of peak average recovery rate and fastest average recovery rate. With respect to reaching a 50\% recovery rate, the $1 \mathrm{WCI}$ group also required the least mean average number of days of

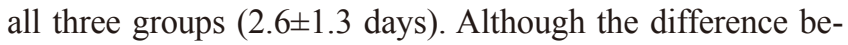
tween 1WCI group and RP group was not statistically significant, we could infer that the use of $\mathrm{CI}$ as a treatment protocol for reconstruction of a mandibular defect could accelerate the speed of rehabilitation better than the RP system. This finding could be explained by the fact that the CIs were created with the same anatomical characteristics as the mandibular defect through the combination of $\mathrm{CBCT}$ and $\mathrm{CAD} / \mathrm{CAM}$ technology. Moreover, the advantage of 3D printing enables the possibility of creating porous structures from a metallic material to mimic original bone ${ }^{23}$. The result is an ideal CI designed with an almost identical external shape to that of the mandibular defect and containing a porous internal structure to enhance bone ingrowth.

The $1 \mathrm{WCI}$ group required $2.6 \pm 1.3$ days to reach $50 \%$ recovery, while the $6 \mathrm{WCI}$ group required $7.8 \pm 2.3$ days. There was a statistically significant difference between these two groups $(P=0.001)$. As mentioned above, the difference between these subgroups was that the $6 \mathrm{WCI}$ group used the CBCT data taken six weeks before surgery for the manufacturing of the CIs, while the CBCT data used for the same in the $1 \mathrm{WCI}$ group were taken at one week before the surgery. It should also be noticed that the $6 \mathrm{WCI}$ group was the only group that had missing screws according to plain radiography review at monthly intervals and at sacrifice. Coincidentally, two rabbits in the 6WCI groups, which had missing screws, also showed signs of infection and plate exposure. At sacrifice, one of them even showed instability of the CI. However, no signs of complication were observed in the 1WCI group. It could be suggested from this finding that the $\mathrm{CBCT}$ data used for the manufacturing of $\mathrm{CI}$ could have an essential role in treatment protocols using CIs as means of reconstruction. The time factor could affect the outcome of this treatment procedure. More recent CBCT data will provide a better foundation for more accurate CIs. In this study, $6 \mathrm{WCI}$ groups used the CBCT data collected at six weeks before the operation. The length of time, in conjunction with the fast-growing rate of rabbits, made these $\mathrm{CBCT}$ data inappropriate for the manufacturing of CIs in this group. This factor should be considered when using CIs for reconstruction surgery in a human. In cases of mandibular defects related to tumors, the growth rate of the tumor or the recurrence of a tumor could affect the size of the defect ${ }^{24}$, leading to a mismatch of the designed $\mathrm{CI}$ and the defect. The timing of CBCT data collection should be selected carefully to ensure a more precise evaluation of the defects is completed so as to increase the accuracy of CIs for reconstruction of the particular defect.

The screw-loosening rate of the $1 \mathrm{WCI}$ group was the lowest among all of the groups. In a related fashion, when comparing the intact screw rate, the 1WCI group showed a higher percentage of such than the RP group. It is possible that the mandibular morphogenesis was influenced by a series of stress factors induced masticatory muscle forces ${ }^{25}$. The shape of the mandible is suitable for the distribution of occlusal forces in different horizontal mandibular positions. The appearance of a mandibular defect may decrease the masticatory efficiency of a patient because of discontinuation of the mandible ${ }^{26}$. Another factor able to contribute to stress distribution is the degree of surface contact between the reconstruction replacements and the bony segments of the mandible. A higher degree of surface contact will reduce stress concentrated on the reconstruction replacements and improve their stability, which has been reported in a previous comparison study between carbon plates and $\mathrm{RPs}^{27}$. In mandibular reconstruction using the RP system, the interface between the plate and screws is usually put under stress created by masticatory movement ${ }^{28}$. Continuous stress on the plate and screws could be one of the reasons for screw loosening, which has already been reported in prior research ${ }^{29}$. To avoid stress concentration, the screw alignment should be designed in a nonlinear formation, which is hardly possible in an RP system ${ }^{30}$. In the present study, the CIs were designed with a mesh structure connected by a titanium plate. The CI ultimately fulfilled three factors that could reduce stress concentration: (1) The shape of the CI mimicked the original defect to recreate the continuous span of the mandible; (2) the CI had a wider contact area with the bony segments compared with an RP; and (3) the screw formation in the CI was designed to appear in a zig-zag formation, which enabled equal stress distribution between screws.

Despite the advantages of CIs compared with RPs as mentioned above, screw loosening was still observed in the CI groups at a relatively low rate, suggesting a slight degree of mismatch between the CIs and the mandibular defect, even 
in the 1WCI group. There could be an explanation for this finding. Incorrect positioning or design of the surgical guide could lead to incorrect osteotomy ${ }^{31}$. These errors could occur during planning procedures or the surgery itself. Examples of errors in planning procedures could be the use of inappropriate CBCT data or overlapping the CBCT data and 3D images. Errors during surgery can happen if the guide is not placed accurately or the guide is moved due to unwanted forces during the osteotomy. There have been many studies emphasizing the importance of using a precise surgical guide in the conduct of surgery based on CAD/CAM technology ${ }^{16}$. To prevent such errors, the CBCT data should be evaluated carefully for better fabrication of both the surgical guide and $\mathrm{CI}$, as mentioned above. The surgical guide could also be designed with landmark indicators such as the host's teeth for precise placement or could include fixation holes for better stability during osteotomy.

In this study, some but not all CIs allowed bony ingrowth into the porous structures. To allow for bony ingrowth, initial stability of the CI must be achieved immediately following CI fixation, which is similar to the case of dental implant osseointegration $^{32}$. Intermaxillary fixation could be an additional procedure performed during the initial stage of recovery to enhance the stability of $\mathrm{CI}$ by avoiding masticatory movement. However, this procedure may be impossible to complete in an animal experiment. A soft diet could also be recommended during the recovery stage to reduce high masticatory forces. However, a prescribed change of diet could affect the DFIA pre- and postoperation, which eventually may lead to errors in calculating the recovery rate.

The 1WCI group showed better efficiency in terms of the recovery rate compared with the RP group. However, the difference was not statistically significant, which could be because the use of DFIA as the only indicator of recovery rate affected the statistical analysis. Another indicator such as average body weight should also be considered to more accurately determine the difference among groups.

Achieving dental rehabilitation was not the main aim of this research. Future designs for CIs should consider the inclusion of dental implants as an additional goal before being applied in fully rehabilitating patients.

\section{Conclusion}

In conclusion, rabbits with mandibular defects treated using CIs for reconstruction showed higher and faster recovery rates of their DFIA than those treated with five-hole mini-plates without bone grafts. However, this could only be achieved with precise utilization and careful evaluation based on more recent CBCT data. The screw status showed favorable results in the CI group, which showed higher screw intact and lower screw loosening rates compared with the RP group. The CI group showed bony ingrowth into the porous structure; however, precise fabrication and placement of the surgical guide for the planned position were required for accurate osteotomy in the CI group. Future design of CIs should require consideration of dental implants in fully rehabilitating patients.

\section{ORCID}

Khang Do Gia Hong, https://orcid.org/0000-0002-3090-724X

Seong-Gon Kim, https://orcid.org/0000-0001-5088-2732

Young-Wook Park, https://orcid.org/0000-0001-5881-7257

\section{Authors' Contributions}

K.D.G.H. performed animal experiment and wrote the first draft of the manuscript. S.G.K. participated in the study design and performed the statistical analysis. Y.W.P. supervised the animal experiment and approved the final manuscript.

\section{Ethics Approval and Consent to Participate}

This experiment was approved by the Institutional Animal Care and Use Committee of Gangneung-Wonju National University, Gangneung, Republic of Korea (GWNU-201625).

\section{Conflict of Interest}

No potential conflict of interest relevant to this article was reported.

\section{References}

1. Standring S. Gray's anatomy: the anatomical basis of clinical practice. 40th ed. London: Churchill Livingstone; 2008.

2. Genden EM, Kessler SM. Reconstruction of the head and neck: a defect-oriented approach. New York: Thieme; 2012.

3. Rachmiel A, Shilo D, Blanc O, Emodi O. Reconstruction of complex mandibular defects using integrated dental custom-made titanium implants. Br J Oral Maxillofac Surg 2017;55:425-7.

4. Millard DR Jr, Deane M, Garst WP. Bending an iliac bone graft for anterior mandibular arch repair. Plast Reconstr Surg 1971;48:6002.

5. Blocker TG Jr, Stout RA. Mandibular reconstruction, World War 
II. Plast Reconstr Surg (1946) 1949;4:153-6.

6. Ostrup LT, Fredrickson JM. Reconstruction of mandibular defects after radiation, using a free, living bone graft transferred by microvascular anastomose. An experimental study. Plast Reconstr Surg 1975;55:563-72.

7. Hidalgo DA. Fibula free flap: a new method of mandible reconstruction. Plast Reconstr Surg 1989;84:71-9.

8. Kim JW, Hwang JH, Ahn KM. Fibular flap for mandible reconstruction in osteoradionecrosis of the jaw: selection criteria of fibula flap. Maxillofac Plast Reconstr Surg 2016;38:46.

9. Goh BT, Lee S, Tideman H, Stoelinga PJ. Mandibular reconstruction in adults: a review. Int J Oral Maxillofac Surg 2008;37:597605.

10. Mücke T, Müller AA, Kansy K, Hallermann W, Kerkmann H, Schuck N, et al. Microsurgical reconstruction of the head and neck-Current practice of maxillofacial units in Germany, Austria, and Switzerland. J Craniomaxillofac Surg 2011;39:449-52.

11. Chow JM, Hill JH. Primary mandibular reconstruction using the AO reconstruction plate. Laryngoscope 1986;96:768-73.

12. Saunders JR Jr, Hirata RM, Jaques DA. Definitive mandibular replacement using reconstruction plates. Am J Surg 1990;160:387-9.

13. Wei FC, Celik N, Yang WG, Chen IH, Chang YM, Chen HC. Complications after reconstruction by plate and soft-tissue free flap in composite mandibular defects and secondary salvage reconstruction with osteocutaneous flap. Plast Reconstr Surg 2003;112:37-42.

14. Arden RL, Rachel JD, Marks SC, Dang K. Volume-length impact of lateral jaw resections on complication rates. Arch Otolaryngol Head Neck Surg 1999;125:68-72.

15. Spencer KR, Sizeland A, Taylor GI, Wiesenfeld D. The use of titanium mandibular reconstruction plates in patients with oral cancer. Int J Oral Maxillofac Surg 1999;28:288-90.

16. Yu Y, Zhang WB, Liu XJ, Guo CB, Yu GY, Peng X. Three-dimensional accuracy of virtual planning and surgical navigation for mandibular reconstruction with free fibula flap. J Oral Maxillofac Surg 2016;74:1503.e1-10.

17. Rotaru H, Stan H, Florian IS, Schumacher R, Park YT, Kim SG, et al. Cranioplasty with custom-made implants: analyzing the cases of 10 patients. J Oral Maxillofac Surg 2012;70:e169-76.

18. Lee UL, Kwon JS, Woo SH, Choi YJ. Simultaneous bimaxillary surgery and mandibular reconstruction with a 3-dimensional printed titanium implant fabricated by electron beam melting: a preliminary mechanical testing of the printed mandible. J Oral Maxillofac Surg 2016;74:1501.e1-15.

19. Cheng G, Li Z, Wan Q, Lv K, Li D, Xing X, et al. A novel animal model treated with tooth extraction to repair the full-thickness defects in the mandible of rabbits. J Surg Res 2015;194:706-16.

20. Chung SM, Jung IK, Yoon BH, Choi BR, Kim DM, Jang JS. Evaluation of different combinations of biphasic calcium phosphate and growth factors for bone formation in calvarial defects in a rabbit model. Int J Periodontics Restorative Dent 2016;36 Suppl:s49-59.

21. Grünheid T, Brugman P, Zentner A, Langenbach GE. Changes in rabbit jaw-muscle activity parameters in response to reduced masticatory load. J Exp Biol 2010;213:775-81.

22. Moon YM, Kim YJ, Kim MK, Kim SG, Kweon H, Kim TW. Early effect of Botox-A injection into the masseter muscle of rats: functional and histological evaluation. Maxillofac Plast Reconstr Surg 2015;37:46.

23. Dawood A, Marti Marti B, Sauret-Jackson V, Darwood A. 3D printing in dentistry. Br Dent J 2015;219:521-9.

24. Chae MP, Smoll NR, Hunter-Smith DJ, Rozen WM. Establishing the natural history and growth rate of ameloblastoma with implications for management: systematic review and meta-analysis. PLoS One 2015;10:e 0117241.

25. Ishida T, Soma K, Miura F. [Stress distribution in mandible induced by occlusal force in different horizontal mandibular positions]. Nihon Kyosei Shika Gakkai Zasshi 1988;47:767-79. Japanese.

26. Namaki S, Matsumoto M, Ohba H, Tanaka H, Koshikawa N, Shinohara M. Masticatory efficiency before and after surgery in oral cancer patients: comparative study of glossectomy, marginal mandibulectomy and segmental mandibulectomy. J Oral Sci 2004;46:113-7.

27. Lee SW, György S, Choi JB, Choi JY, Kim SG. Carbon plate shows even distribution of stress, decreases screw loosening, and increases recovery of preoperative daily feed intake amount in a rabbit model of mandibular continuity defects. J Craniomaxillofac Surg 2014;42:e245-51.

28. Pinheiro M, Alves JL. The feasibility of a custom-made endoprosthesis in mandibular reconstruction: Implant design and finite element analysis. J Craniomaxillofac Surg 2015;43:2116-28.

29. Cohen A, Laviv A, Berman P, Nashef R, Abu-Tair J. Mandibular reconstruction using stereolithographic 3-dimensional printing modeling technology. Oral Surg Oral Med Oral Pathol Oral Radiol Endod 2009;108:661-6.

30. Davidson J, Birt BD, Gruss J. A-O plate mandibular reconstruction: a complication critique. J Otolaryngol 1991;20:104-7.

31. Jo C, Bae D, Choi B, Kim J. Removal of supernumerary teeth utilizing a computer-aided design/computer-aided manufacturing surgical guide. J Oral Maxillofac Surg 2017;75:924.e1-9.

32. Lee JW, An JH, Park SH, Chong JH, Kim GS, Han J, et al. Retrospective clinical study of an implant with a sandblasted, large-grit, acid-etched surface and internal connection: analysis of short-term success rate and marginal bone loss. Maxillofac Plast Reconstr Surg 2016;38:42.

How to cite this article: Hong KDG, Kim SG, Park YW. The effect of fixation plate use on bone healing during the reconstruction of mandibular defects. J Korean Assoc Oral Maxillofac Surg 2019;45:276-284. https://doi.org/10.5125/jkaoms.2019.45.5.276 